\title{
MASS LOSS AND CEPHEID PULSATION
}

\author{
Cecil G. Davis, Jr. \\ U. of California, Los Alamos Scientific Laboratory \\ Los Alamos, New Mexico, USA
}

\begin{abstract}
This paper serves two purposes: 1) to discuss the latest improvements in nonlinear pulsation theory indicating the ability to resolve features such as the "Christy bump" on the light curves and 2) to show from the results of a bump model and recent observations that mass loss is one of the possible explanations for the mass discrepancy problem between evolutionary and pulsation theories. Recent observations by Sanford and Gow of Los Alamos and Bernat (McDonald Observatory) show that extensive mass loss has occurred in the evolution of the M supergiant $\alpha$ orionis.
\end{abstract}

\section{Introduction}

By using a new dynamic zoning technique and the variable Eddington radiative transfer approximation in a nonlinear pulsation code, coupled with new low temperature opacities, we have produced a light curve and colors that can now be compared directly to the observations. Previous nonlinear models (Christy - 1968 and Stobie1969) using Lagrangian hydrodynamic and radiative diffusion relied on the comparison of the calculated velocities to those inferred from the observation of lines for the determination of the mass due to the location of the bump. In Sec. II we describe the various suggestions put forth to explain the mass discrepancy problem. The "Goddard" model, a model of a 10-day Cepheid with a bump that had been studied extensively, is described in sec. III. This model closely approximates the parameters of the observed galactic cepheid $S$ Nor and direct comparisons of the light and colors are made in sec. IV. The conclusion is that with the new modeling techniques, light curves can be calculated that will compare in detail with the observation, but again (Christy - 1968) showing that the location of the bump depends on the assumption of mass loss. The recent observations of extensive mass loss for stars in the giant branch of stellar evolution are discussed in Sec. V. These results would support our contention (Sec. IV) that mass loss is needed in stellar models that evolve into the instability strip using Cox-stewart opacities. 


\section{Mass Discrepancy}

We begin by discussing the various ideas that have been proposed to explain the mass discrepancy problem. The discrepancy that exists is between the mass evolved theoretically into the cepheid instability strip and the mass necessary to explain the constraints due to pulsation theory, or the mass due to the location of the "bumps" observed in the Hertzsprung sequence. Most of these ideas have been discussed in the article by Fricke, stobie and strittmatter (1972).

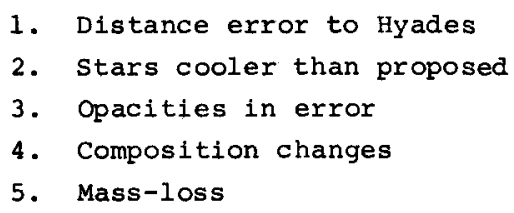

1) Iben and Tuggle (1972) suggest that the luminosity of Cepheids should be increased because the distance modulus to the Hyades is low by $0.3 \mathrm{mag}$, (Hodge and Wallerstein - 1966). This suggestion is supported by some recent work of Twarog and Demarque (1977) and it removes the discrepancy in the $\mathrm{P}-\mathrm{L}-\mathrm{M}$ relationship.

2) That the conversion from $(B-V)$ to $T_{\text {eff }}$ should result in stars cooler by 300 to $600^{\circ} \mathrm{K}$ for the hotter stars. This idea appears to be unacceptable since a large gap would occur between the calculated and observed blue edges.

3) Recent new opacity calculations by Carson (1976), utilizing the "hot" Thomas Fermi model for the atom, have produced large increases in opacity in the region of CNO lonization and some differences also appear in the hydrogen ionization region around $10000-12000^{\circ} \mathrm{K}$. Estimates by Carson and stothers (1976) and recent direct nonlinear numerical work described by Vemuri and stothers (1977)-(S.K. Vemury and R. Stothers in Abstracts of 150th meeting AAS - 1977), apparently shows agreement with the Hertzsprung bumps using the evolutionary mass and the Carson opacities.

4) Probably one of the more exciting ideas is that of Cox (1977) on proposed composition changes near the star's surface. He proposes along the lines of Michaud's research, that separation of atomic constituents would occur due to radiation pressure effects on the Iines, particularly on the helium lines. Linear analysis of models with reasonable composition changes have shown double mode behavior using evolutionary masses (Cox and Deupree - 1977). 
5) The idea that mass loss explains the mass discrepancy was discussed by Tayler (1970) and is strengthened by the recent observation of a large circumstellar shell surrounding Betalgeuse, to be described in Sec. V. Estimates of the effect of large mass loss on stellar evolution were made by Forbes (1968) for a 5-solar mass model. He finds that these stars will evolve into the instability strip.

\section{II.l. The Goddard Model}

The most extensively studied model of a 10-day Cepheid is the so-called "Goddard" model first proposed in 1974 for intercomparison at the Goddard Conference (Fischel and Sparks - 1974) and further studies at the Los Alamos Conference on "Solar and Stellar Pulsation" (Cox and Deupree - 1976). The luminosity used in the Goddard model $\left(L / L_{\odot}=3.19 \times 10^{3}\right)$ is in reasonable agreement with that observed for the galactic Cepheid $S$ Nor $\langle\langle M v\rangle=-4.05)$. The $B-V$ observed for $S$ Nor, corrected by reddening, is 0.75 implying a temperature from the Kraft-Oke relationship of $5685^{\circ} \mathrm{K}$. This is in good agreement with our model temperature of $5700^{\circ} \mathrm{K}$. The model period is 9.8 compared to the observed period for $S$ Nor of 9.75 . The mass used in this model, as in the Christy and stobie models, is only 608 of the evolutionary mass or $4.0 \mathrm{M}$.

The major problem in producing resolved light curves results from the use of finite zone sizes in the Lagrangian mesh. Recently J. Castor has devised a non-Lagrangian or dynamically zoned scheme which we have applied to the Cepheid pulsation problem (Castor, Davis \& Davison - 1977). By treating the mass as an independent variable we are able to keep the Lagrangian zone boundaries in the hydrogen ionization front during the course of a pulsation period. The zone interfaces used in the radiative flux equation, for a particular phase, are shown in Figure 1. By carefully selecting the zones in a typical Lagrangian scheme we are also able to obtain similar results (Figure 2). The advantage at present of using the Lagrangian code is due to the adaptation of the variable Eddington approximation for the radiative transfer. This approximation uncouples the radiative and material energy fields. The results from a sequence of three periods, showing the resolved features in the light curve are shown in Figure 3. The shoulder appearing before light maximum is related to hydrodynamic motion in the atmosphere 
616

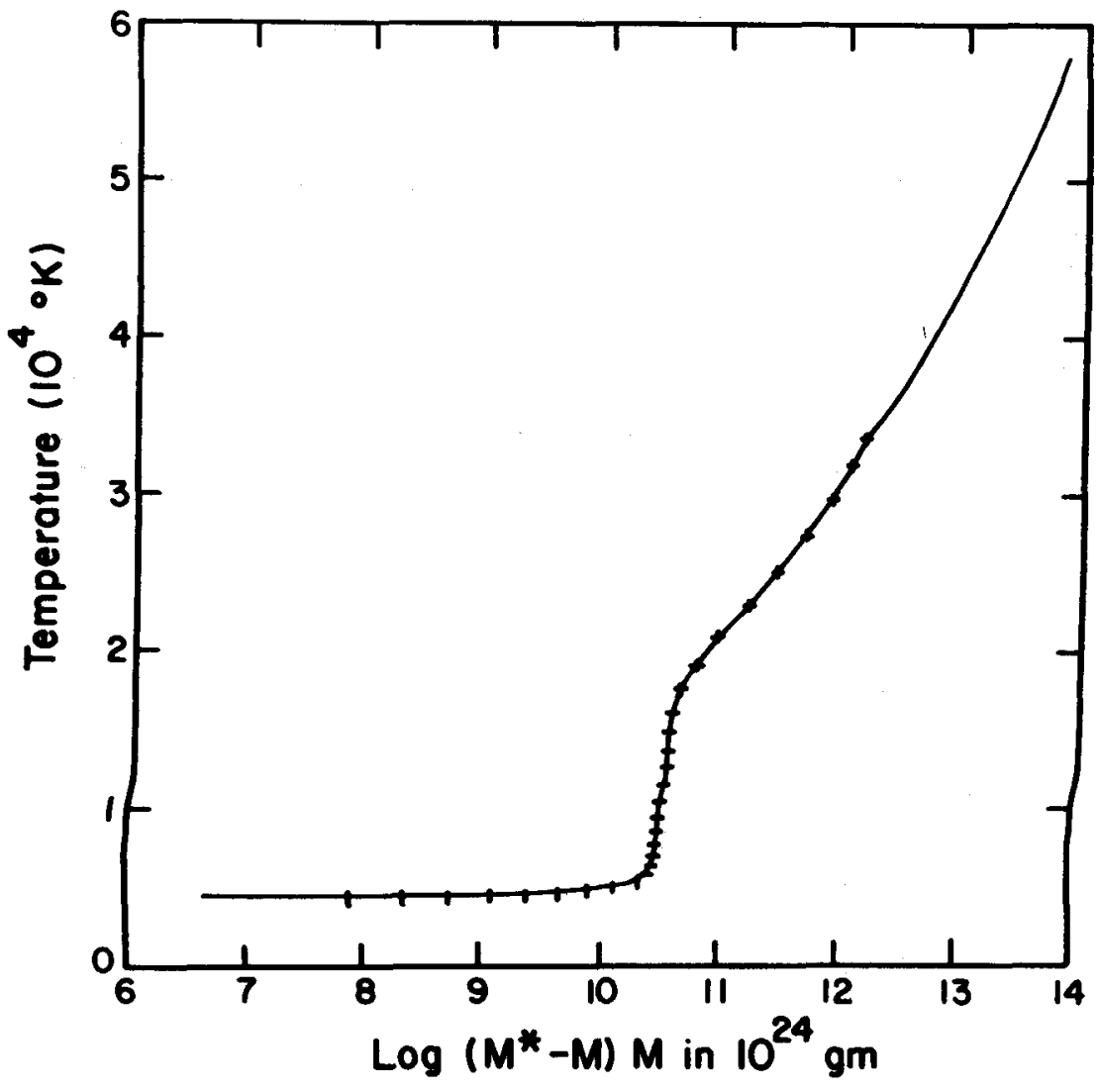

Fig. 1. Typical zoning in the dynamically zoned Goddard model. 


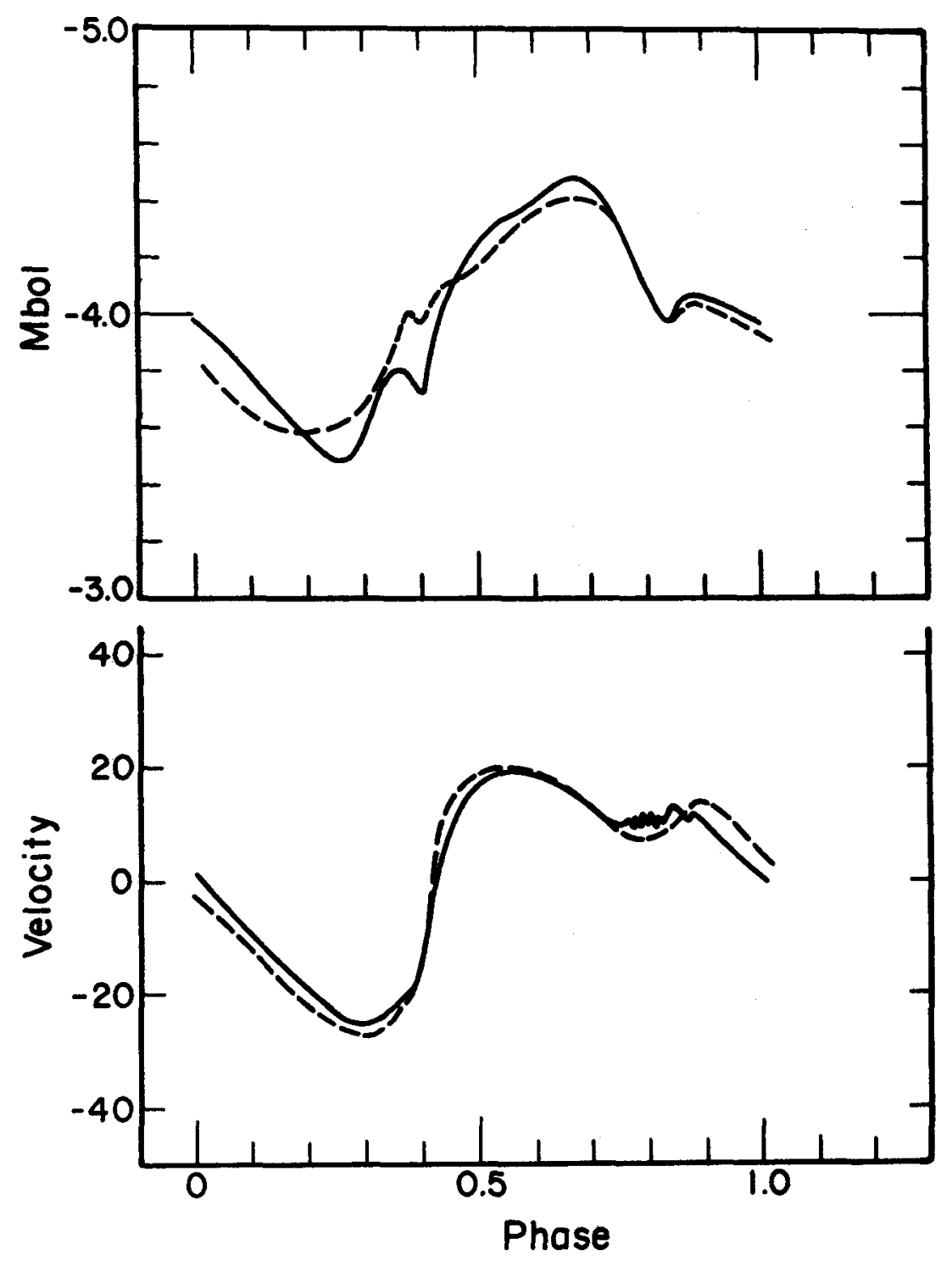

Fig. 2. Limited amplitude Iuminosity and velocity curves. DYN (solid line), SPEC results (dashed line). 
of the star, possibly a shock transiting the atmosphere while the bump after light maximum, which appears at approximately the same phase in the velocity curve, is definitely the result of an echo reflected from the star's interior.

\section{Comparison to Observations}

As mentioned in Sec. II, the Goddard model has a luminosity and color that is close to that observed for the Cepheid $s$ Nor. In Fig. 3 is seen a smoothed reduction of the data from Breger (1970) for $S$ Nor. There is a definite shoulder before light maximum, as is also observed for the 10-day cepheid $\beta$ Doradus, which appears to correlate with an increase in UV luminosity as observed by . Hutchinson (1974). This UV radiation bump again appears at the time that a shock is propagating through the atmosphere of the Goddard model. At present, however, there is no conclusive evidence for shocks occurring in cepheids as is observed for $W$ Virginis and RR Lyrae stars (line splitting). The "bump" occurring after light maximum is more clearly resolved in the data from $\beta$ Doradus (Hill 1976).

As further evidence that this model correlates well with the observations, we have calculated the colors using a blanketed set of opacities with a slightly different composition ( $Y=0.28, z=0.02)$. The increased zoning through the hydrogen ionization region results in an improved calculation of the $U, B, V$ colors, especially for the ultraviolet $(U)$. The $B-V$ color difference versus the $\log T$ eff is shown in Fig. 4. The displacement from the Kraft-oke line results from not including these low temperature opacities in the hydrodynamic model (Cox and Davis - 1974). The results of a calculated color-color loop as compared to the observations on $S$ Nor Fernie (1961) are shown in Fig. 5.

\section{Direct Evidence of Mass Loss}

Recent observations carried out at the McDonald Observatory (Bernat - 1977, Sanford - 1977) show that the M supergiant $\alpha$ Orionis is losing mass at an astounding rate. The estimate made by Bernat (1977) of the mass-loss rate for this $M$ supergiant is $3.4 \times 10^{-5}$ $\mathrm{M}_{\odot} / \mathrm{yr}$. This is well within the range needed to explain the mass discrepancy in Cepheid pulsation. Photographs taken on the McDonald 


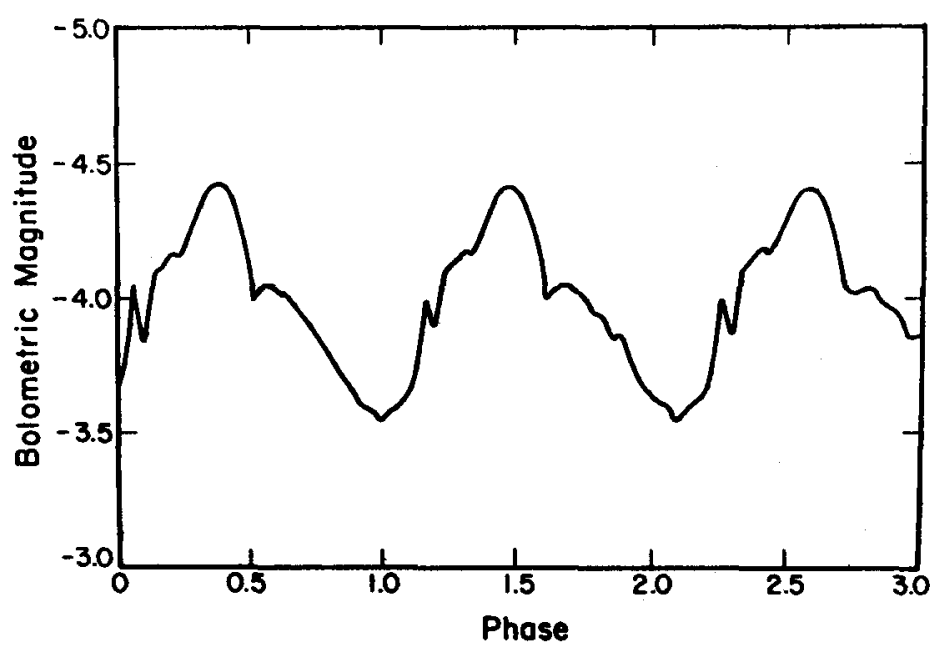

Fig. 3. Calculated light curve showing repetition of calculated features at limiting amplitude.

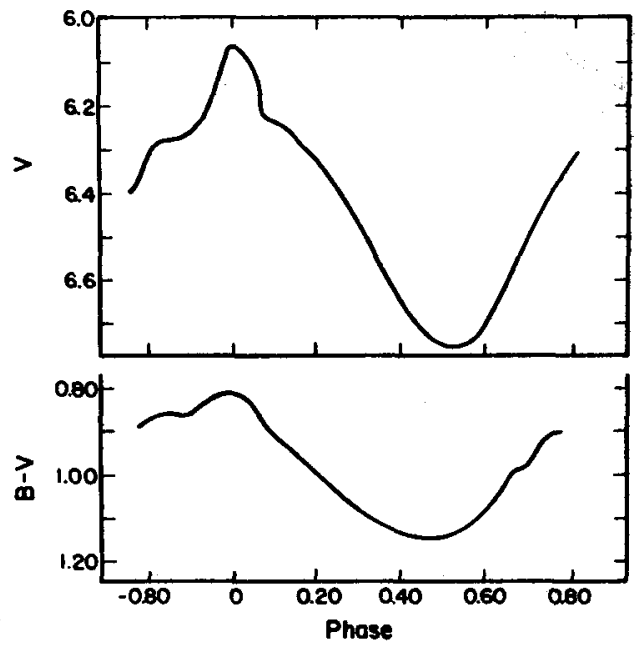

Fig. 4. The observed light and color curves for the Cepheid $s$ Nor, uncorrected for reddening (Fernie, 1966). 


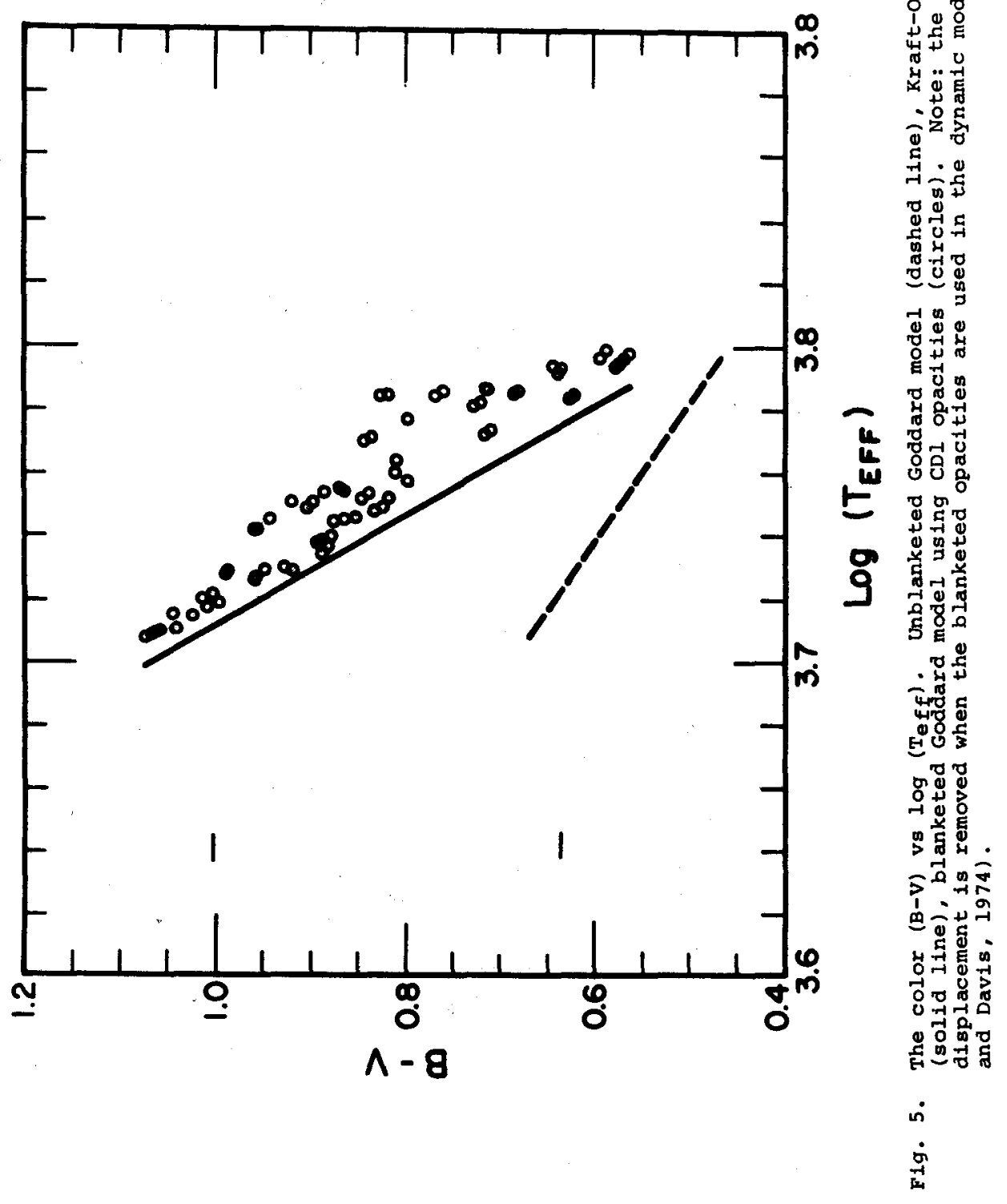



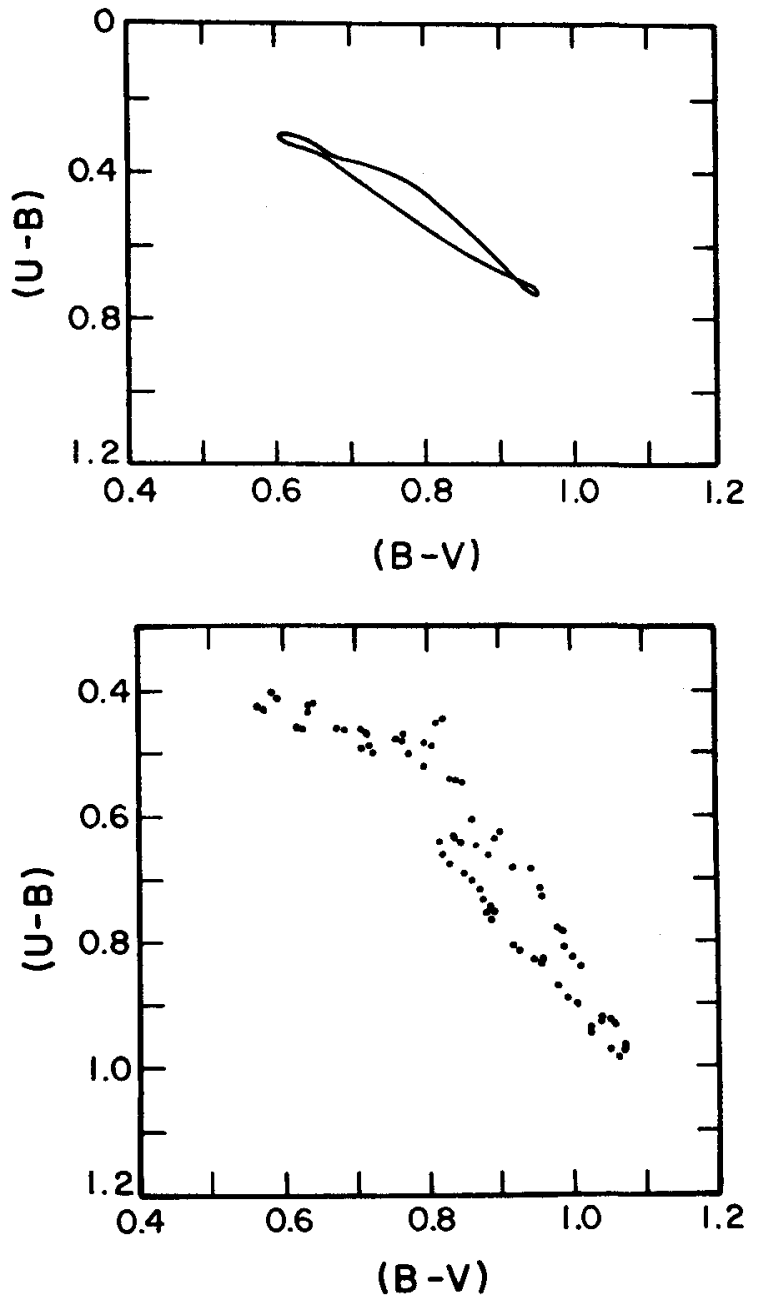

Fig. 6. The observed (above) and calculated (below) color-color loops for the Cepheid $S$ Nor. 
82" telescope, using a SIT (IR TV tube) by Sanford (1977) show the extent of the circumstellar shell surrounding Betelgeuse. These are photogrpahs of the neutral potassium line and the off line sky background for comparison. The extent of the shell is approximately 400 solar system diameters. Forbes (1968) showed that by using a rate like $10^{-6} / \mathrm{M}_{\Theta} / \mathrm{Yr}$ for his low mass star $\left(5 \mathrm{M}_{\Theta}\right)$ more than $80 \%$ of the mass was lost by the time the star reached the instability strip with only a slight change in the luminosity. A 7-solar mass star with the observed mass loss rate could then quite easily evolve into the instability strip reaching a 608 reduction in mass as needed by pulsation theory.

\section{Conclusions}

It appears that the mass discrepancy problem in pulsation theory can be explained by various means. Recent support by Twarog and Demarque (1977) for the distance correction to the Hyades makes this suggestion very attractive for a solution to the $\mathrm{P}-\mathrm{L}-\mathrm{M}$ relationship using evolutionary masses. Apparently one can throw out the suggestion that the temperature-color transformation should produce cooler stars. The discrepancy with the observed blue edge seemingly rules out this suggestion.

On the question of recent opacity changes, again there is no consensus on whether the hot "Thomas Fermi" approximateion or the hydrogenic approximations are the appropriate ones, but as yet the new Carson opacities are unpublished. The proposition that composition changes occur is an interesting idea based on fairly complex physics that has yet to be understood completely (Cox - 1977). The Idea has improved our interpretation of double mode cepheid masses (Cox and Deupree - 1977) and may possibly remove the "bump" Cepheld mass discrepancy problem. From our interpretation of the recently improved light curves for these lower-than-evolutionary mass 10-day Cepheids, it is possible that mass loss is an alternative explanation. The recent observations of large mass loss from $\alpha$ orionis supports this position.

\section{References}

Bernat, A.P. 1977, Ap. J., 213, 756.

Breger, M. 1970, The Astron. J. 75, 239. 
Carson, T.R. 1976, Ann. Rev.Astrophysics, p. 95.

Carson, T.R. and Stothers, R. 1976, Ap. J., 204, 461.

Castor, J.J., Davis, C.G. and Davison, D.D. 1977, (DYN), Los Alamos

Scientific Laboratory Report, LA-6644.

Christy, R. 1968, Quart. J.R.A.S., 9, 13.

Cox, A.N. and Deupree, R. 1976, Solar and Stellar Pulsation

Conference, Los Alamos Scientific Laboratory Report, LA-6544C.

Cox, A.N. and Deupree, R. 1977, Ap. J. , 214, L 127.

Cox, A.N. 1977, Private communication.

Fernie, J.P. 1966, Ap. J., 133, 64.

Fischel, D. and Sparks, W. 1974, Cepheid Modeling, NASA-SP-383.

Forbes, J.E. 1968, Ap. J., 153, 495.

Fricke, K., Stobie, R.S. and Strittmatter, P.A. 1972, Ap. J., 171, 593.

Hill, S.J. 1976, Solar and Stellar pulsation Conference, Los Alamos Scientific Laboratory Report, LA-6544C, 265.

Hodge, F.W. and Wallenstein, G. 1966, PubI. Astron. Soc. Pac., 78, 411.

Hutchinson, J.L. 1974, Cepheid Modeling, NASA-SP-383, 5.

Iben, I., Jr. and Tuggle, R.s. 1972, Ap. J., 173, 135.

Sanford, M.T. 1977, Private communication.

Stobie, R.S. 1969, M.N.R.A.S., 144, 461.

Tayler, R.J. 1970, M.N.R.A.S., 149, 17.

Twarog, B.J. Anthony and Demarque, P. 1977, Astron. Astrophys. 57, 471 .

DISCUSSION of paper by DAVIS:

PEL: I have two questions: 1) what are your comments on the results by Lauterborn, Refsdal, and others who showed that with more than loz mass loss in the red giant stage, the loops in the evolutionary tracks do not extend into the cepheid region any more.

2) I do not quite understand your statement that adopting lower temperatures for the Cepheids - which would diminish or even solve the mass discrepancy - would be inconsistent with the position of the blue edge. The equilibrium Cepheid temperatures that I get from my photometer after calibrating with the model spectra of Kurucz, produce a beautiful fit to the blue edge, but are up to a few hundred degrees cooler than temperatures adopted up to now. In view of the many uncertainties in the observed 
quantities and their translation into theoretical parameters, I feel that the theoreticians may have been worried too much about the Cepheid mass problem.

DAVIS: 1. It is possible that effects of semi-convection or rotation may affect the evolutionary tracks.

2. It would be exciting if the lower temperatures of the (B-V) conversions work.

KIPPENHAHN: I would like to ask Prof. Renzini what deviations from the (constant mass) evolutionary calculations he obtains for Cepheid masses if he takes Reimers' mass loss formula.

RENZINI: The time spent as a red ${ }_{4}$ giant before the cepheid phase is so short that only $10^{-3}-10^{-4} \mathrm{M}_{0}$ are lost (using the Reimers mass loss rate). Even using the Bernat mass loss rate the situation does not change appreciably. On the other hand, if one insists that intermediate mass stars lose $\sim 208$ of their mass on the red giant branch before the Cepheid phase, one finds exceedingly too large mass losses during the red giant branch phase of low mass stars and during the second rise to the red giant branch (double-shell models). In this case horizontal branch stars would disappear (low mass stars would fail to ignite helium) and Mira variables would also disappear (the second red giant branch phase would be dramatically shortened).

To avoid such disastrous implications I believe one should find the solution of the so-called Cepheid mass discrepancy in something else than mass loss.

WEYMANN: zuckermann has a preliminary detection of the cloud around $\alpha$ Ori in $21 \mathrm{~cm}$ which suggests $M \approx$ those derived by Reimers and others.

KRAFT: I suggest that you use the new temperature scales of Dr. Pel which are based on Kurucz' blanketed model atmospheres, which are much more reliable than our old temperatures bases essentially on the MK temperature scale.

GEYER: One intriguing problem to me is the position of very rare stars 2-4 magnitudes above the HB of globular clusters. Here also occur the RV Tauri variables. Do you have any idea which mechanism in contrast to the HB stars brings them into this position of the c-m-diagram?

DAVIS: Sorry, no. 